\title{
Dermoscopy Update: Review of Its Extradiagnostic and Expanding Indications and Future Prospects
}

\author{
Sidharth Sonthalia ${ }^{1}$, Paola Pasquali ${ }^{2}$, Mahima Agrawal $^{3}$, Poonam Sharma ${ }^{4}$, \\ Abhijeet K. Jha ${ }^{5}$, Enzo Errichetti ${ }^{6}$, Aimilios Lallas ${ }^{7}$, Virendra N. Sehgal ${ }^{8}$
}

\begin{abstract}
1 SKINNOCENCE: The Skin Clinic \& Research Centre, Gurugram, India
2 Department of Dermatology, Pius Hospital de Valls, Tarragona, Spain

3 Department of Dermatology \& STD, LHMC \& Associated Hospitals, New Delhi, India

4 Skin Institute \& School of Dermatology (SISD), New Delhi, India

5 Department of Dermatology \& STD, Patna Medical College \& Hospital, Patna, India

6 Institute of Dermatology, University Hospital Santa Maria della Misericordia, Udine, Italy

7 First Department of Dermatology, Aristotle University, Thessaloniki, Greece

8 Department of Dermatology, Sehgal Nursing Home, New Delhi, India
\end{abstract}

Key words: dermoscopy, trichoscopy, onychoscopy, clinico-dermoscopic correlation, dermatopathology-dermoscopic correlation, teledermoscopy

Citation: Sonthalia S, Pasquali P, Agrawal M, Sharma P, Jha AK, Errichetti E, Lallas A, Sehgal VN. Dermoscopy update: review of its extradiagnostic and expanding indications and future prospects. Dermatol Pract Concept. 2019;9(4):253-264. DOI: https://doi.org/10.5826/ dpc.0904a02

Accepted: July 7, 2019; Published: October 31, 2019

Copyright: $@ 2019$ Sonthalia et al. This is an open-access article distributed under the terms of the Creative Commons Attribution License, which permits unrestricted use, distribution, and reproduction in any medium, provided the original author and source are credited.

Funding: None.

Competing interests: The authors have no conflicts of interest to disclose.

Authorship: All authors have contributed significantly to this publication.

Corresponding author: Sidharth Sonthalia, MD, DNB, MNAMS, Skinnocence: The Skin Clinic \& Research Centre, C-2246, Sushant Lok-1, Block-C, Gurugram 122009, India. Email: sidharth.sonthalia@gmail.com

ABSTRACT The technique of dermoscopy has come a long way since its inception for characterization of suspicious nevi for early detection of dysplastic changes in predisposed individuals. Not only has its scope expanded to aid in quick diagnosis of a majority of nonmelanocytic disorders of the skin, hair, and nails, but it is being rightfully exploited for a plethora of nondiagnostic uses. Its use in the diagnosis of various pigmentary, papulosquamous, and infectious disorders and disorders of the scalp and hair, nails, and mucosa bears testimony to the ongoing expansion of its protean indications across skin types. Dermoscopy has transformed the conventional approach to dermatological diagnosis from clinicopathological correlation to clinico-dermoscopic-pathological correlation. It aids in convincing an otherwise reluctant patient to agree to biopsy and guides the selection of optimum site for the same. Dermoscopic clues suggestive of stability or activity of the lesion and/or disease in various dermatoses are being accrued. Early assessment of therapeutic response to treatment is helpful for physicians, patients, and researchers conducting clinical studies. Aesthetic uses of dermoscopy are opulent and being explored. Dermoscopy has also provided the much-needed balancing act of interaction between practitioners and the new generation of patients. Last but not the least, dermoscopy has resulted in patients' better understanding of their disorders and improved compliance with treatment protocols. 


\section{Introduction}

Since the inception of dermoscopy, hitherto envisaged to evaluate suspicious nevi and noninvasively detect changes in melanocytic lesions of white-skinned individuals, the technology and its applications have come a long way. The diagnostic scope has expanded to practically every disorder of the skin and its appendages. The technology is now being rightfully exploited for its additional nondiagnostic uses [1,2]. Internet and social media have induced a huge change in patients' attitude toward health care. Dermatologists are confronted with the challenge of treating a wider spectrum

Table 1. Gross Estimate of the Trend of Publications Addressing "Dermoscopy" and "Dermatoscopy"

\section{Time Period}

No. of Publications ${ }^{a}$

\begin{tabular}{|l|c|}
\hline Jan 2015-Dec 2017 & 1,173 \\
\hline Jan 2012-Dec 2014 & 2,029 \\
\hline Jan 2009-Dec 2011 & 1,010 \\
\hline Jan 2006-Dec 2008 & 574 \\
\hline Jan 2003-Dec 2005 & 268 \\
\hline
\end{tabular}

aThe figures (number of publications) have been derived from a single portal (www.ncbi.nlm.nih.gov/pubmed/), using the search key words "dermoscopy" and "dermatoscopy" and applying timed filter under "publication dates."

\section{Table 2. Major Extradiagnostic Applications of Dermoscopy}

Dermoscopy and Skin Biopsy

- Patient counseling, dermoscopy-induced biopsy, and the psychological realm of the dermatoscope, akin to the stethoscope of an internist

- From clinicopathological correlation to clinico-dermoscopic-pathological correlation

- Dermoscopy-guided biopsy and the role of ex-vivo dermoscopy in processing of histopathological samples

Dermoscopy as a Diagnostic Enhancer and Monitoring Tool

- Defining lesional or disease activity/instability

- Defining adequate margins for surgical excision of tumors

- Early assessment of therapeutic response and disease stabilization

- Enhancing accuracy of reading patch test and pathergy test results

- Therapeutic efficacy evaluation in clinical studies

Expanding the Realm of Dermoscopy Applications

- "Cosmetic wart mapping"

- Aesthetic applications of dermoscopy

- Detection of intra/subcutaneous foreign bodies and retained sutures

Teledermoscopy

Psychosocial Significance

- The dermatologist's tool in the era of social media empowerment of patients

- A professional highlight for dermatologists

- The dermatologist's stethoscope

\section{Impact of the Publication Spree}

Publications in indexed journals addressing "dermoscopy"/"dermatoscopy" have shown a constant upward trend as depicted in Table 1. Although we employed a rather crude method for these statistics (explained in Table 1 footnotes), the figures offer a fair hint about the increase in awareness with regard to using and publishing about this technique.

\section{Diagnostic Applications of Dermoscopy: Advancing and Evolving}

In the past few years, this technique has been successfully explored for diagnosis of various skin, scalp, and nail disorders [3]. Subspecialties such as pigmentaroscopy, inflammoscopy (papulosquamous disorders), entodermoscopy (infections and infestations), trichoscopy (scalp and hair), onychoscopy (nails), and mucoscopy are branching out within the broad ambit of dermoscopy. Although histopathology remains the current gold standard of cutaneous diagnosis, dermoscopy and other noninvasive techniques have revolutionized the approach to diagnosis of cutaneous disorders. Dermoscopy has expanded from its original use (observation-pattern recognitiondiagnosis) to other applications listed in Table 2.

\section{Dermoscopy and Skin Biopsy}

Patient Counseling, DermoscopyInduced Biopsy, and the Psychological Realm of the Dermatoscope: Stethoscope of the Dermatologist

Skin biopsy, despite its exigency in many situations, is sometimes refused by the patient on various premises. Facial lesions assume a special significance in this context. Despite the appearance of a biopsy-worthy and otherwise distressing lesion/pigmentation on the face, 


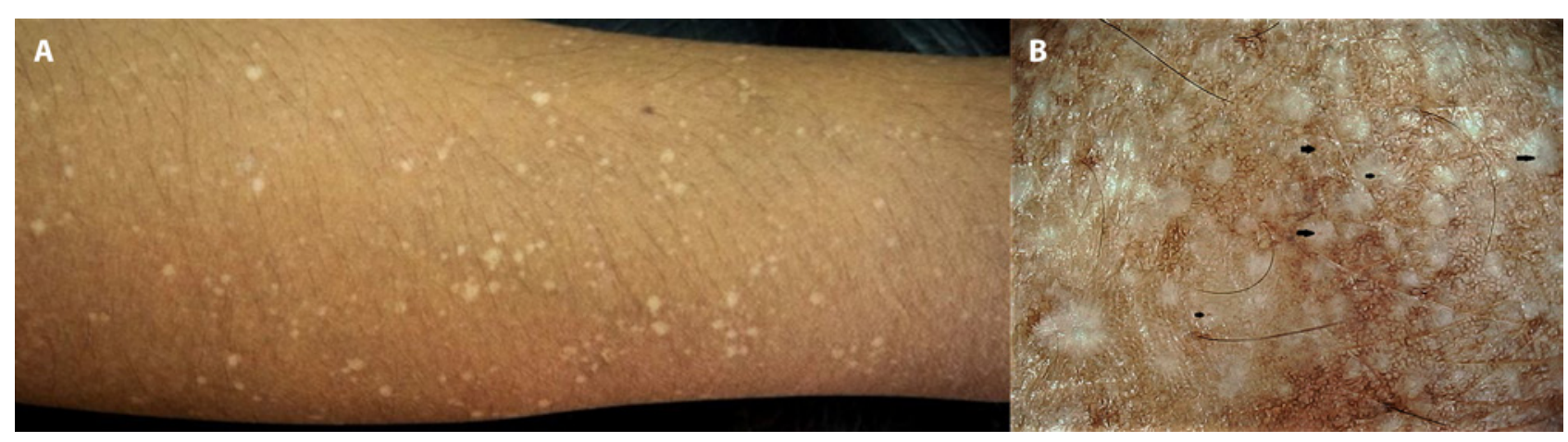

Figure 1. Lesions of idiopathic guttate hypomelanosis and extragenital lichen sclerosus atrophicus over the forearm of a 45 -year-old woman. (A) Clinical picture of multiple discrete "confetti" hypopigmented macules over the forearm that made the patient anxious that she was suffering from vitiligo or leprosy. (B) Dermoscopy confirming the diagnosis of idiopathic guttate hypomelanosis with concomitant evolving lesions of extragenital lichen sclerosus atrophicus. Ameboid and nebuloid variants of idiopathic guttate hypomelanosis arranged in a "cloudy sky" pattern. Additionally, note the scattered white structureless areas with comedo-like plugs typical of lichen sclerosus atrophicus. Dermoscopy-guided biopsy of 2 lesions confirmed dermoscopic diagnosis. (Escope video dermatoscope, Timpac Healthcare Pvt. Ltd., New Delhi, India; ×20.) [Copyright: (C2019 Sonthalia et al.]

facial biopsy is often not acceptable by the patient due to concerns of potential scarring. In such commonly encountered situations, dermoscopy helps in multiple ways.

Used judiciously, dermoscopy can foster doctor-patient communication and improve patient compliance and overall management of the disorder. Demonstration of the dermoscopic image to the patient (as per the dermatologist's discretion) and limited but tactful conversation regarding the visible features can allay the patient's curiosity to understand the pathogenesis of his or her dermatoses. In addition, patients who warrant a skin biopsy but refuse it owing to procedure-related anxiety are more inclined to consent after having been shown the dermoscopic image with emphasis on disease-specific features. In our own experience, since we started using dermoscopy for every patient, we have observed $>90 \%$ success in convincing a reluctant patient to consent to a biopsy, a concept we call "dermoscopy-induced skin biopsy” [1]. Dermoscopy can also be used to reassure a patient who fears having a condition that requires a biopsy and instead has a benign condition, eg, idiopathic guttate hypomelanosis and/or extragenital lichen sclerosus atophicus perceived as vitiligo (Figure 1), or racial benign lon- gitudinal melanonychia being dreaded to suggest subungual melanoma.

Finally, the dermatoscope is in essence the stethoscope of the dermatologist (vide infra). The majority of skin and hair disorders are chronic and recurring, with many being recalcitrant to treatments. Examining a lesion with a dermatoscope and discussing the images with the patient can help in the overall management of the disorder.

\section{From Clinicopathological Correlation to Clinico- Dermoscopic-Pathological Correlation}

Dermoscopists are sometimes faced with situations in which the clinicopathological diagnosis is revised after dermoscopic inputs are considered. For example, a 60-year-old, otherwise healthy man presented with a single, asymptomatic light brown plaque of 2 years' duration over the lower abdomen. Examination revealed a solitary, ovalshaped, 3- $\times$ 4-cm, sharply demarcated annular plaque over the lower right side of the anterior abdomen that had a violaceous-to-brown central region and raised erythematous borders (Figure 2A). Histopathology suggested lichen planus (LP) with mild hyperkeratosis, hypergranulosis with flattening of rete ridges, Max-Joseph space formation, dense bandlike lymphohistiocytic inflammation at the dermoepidermal junction, basal cell degeneration, and colloid bodies (Figure 2B). However, a single lesion, atypical morphology, and lack of mucosal involvement were clinical pointers against LP. Dermoscopic evaluation revealed light brown pseudonetworks, overlapping pinkish areas, and multiple dark brown to blue-gray dots and globules (Figure 2C), with characteristic absence of Wickham striae. The dermoscopic features not only ruled out LP, but were characteristic of an early inflammatory lesion of LP-like keratosis [4]. Thus, an unconvincing clinicopathological diagnosis of LP was revised and confirmed to be LP-like keratosis on clinico-dermoscopic-pathological correlation. Thus, despite histopathology remaining the gold standard for diagnosis of skin disorders, cutaneous diagnosis is trending from clinicopathological correlation to clinico-dermoscopic-pathological correlation [1,2]. Cicatricial alopecias, especially discoid lupus erythematosus (DLE) and lichen plano pilaris, have a considerable overlap in their histology, especially in the late stage of the disease [5-8]. Appendageal loss and replacement with fibrotic tissue, vacuolar degeneration, interface dermatitis, and perifollicular infiltrate are common to both $[6,7]$. Although the inflammatory 


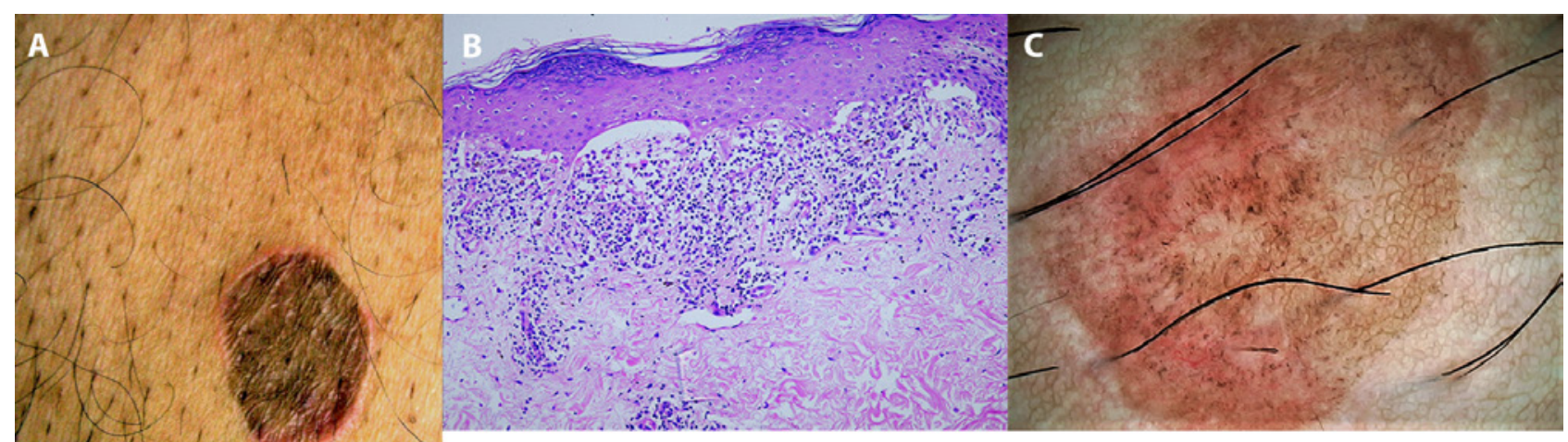

Figure 2. Clinico-dermoscopic-pathological correlation. (A) Single, light brown, oval-shaped annular plaque over the lower anterior abdomen. (B) Histopathology suggestive of lichen planus, revealing mild hyperkeratosis, hypergranulosis with flattening of rete ridges, Max-Joseph space formation, dense bandlike lymphohistiocytic inflammation at the dermoepidermal junction, basal cell degeneration, and colloid bodies (H\&E, $\times 400)$. (C) Dermoscopy revealed light brown pseudonetworks, overlapping pinkish areas, and multiple dark brown to blue-gray dots and globules. Wickham striae were not seen. Final diagnosis of early inflammatory lesion of lichen planus-like keratosis was confirmed. (Escope video dermatoscope, Timpac Healthcare Pvt. Ltd., New Delhi, India; polarized, ×20.) [Copyright: $\odot 2019$ Sonthalia et al.]

infiltrate is deeper in DLE, this feature is often insufficient for a confident diagnosis of DLE. Furthermore, the interpretation of a scalp biopsy sample is highly dependent on the site from which it is obtained. The cost and logistics of direct immunofluorescence often override the technique's utility in differentiation of the 2 conditions. Trichoscopy dramatically simplifies this issue because classic lichen plano pilaris, scalp DLE, and frontal fibrosing alopecia have distinctive trichoscopic features $[6,8]$.

\section{Dermoscopy-Guided Biopsy and the Role of Ex-Vivo Dermoscopy in Histopathology Processing}

The dermatopathologist's efficiency in providing a straightforward, confident diagnosis is highly dependent on various factors from the site selection, adequacy of biopsy sample, and details filled in the pathology requisition form [9]. Dermoscopy helps in selection of the optimum site for biopsy, which increases the likelihood of a definitive histopathological diagnosis instead of reporting of nonspecific features by the pathologist. This concept of "dermoscopy-guided skin biopsy" has been reported to be advantageous in cicatricial alopecias, vasculitis, facial melanosis (eg, patient with melasma with concomitant exog- enous ochronosis), collection of nail scrapings for mycological testing, and skin tumors, among others [10-15]. Dermoscopy has recently been reported to contribute further to the accuracy of histopathological diagnosis. Dermoscopy-guided histological sectioning of the biopsy tissue has been shown to optimize the sectioning of melanocytic neoplasms [15]. This principle of adapted sectioning employing ex-vivo dermoscopy may be combined with derm-dotting (marking a dermoscopically suspicious area of the specimen with nail varnish) to allow more accurate and less time-consuming histopathological diagnosis of skin tumors in a dermatopathology setting [16].

\section{Dermoscopy as a Diagnostic Enhancer and Tool for Monitoring Disease Activity}

\section{Defining Lesional or Disease Activity/Instability}

In chronic dermatoses with a relapsing-remitting course, the disease and/ or lesional activity assumes importance when a specific intervention is being considered. Prototypically, in vitiligo medical treatment remains the primary approach but surgical repigmentation is often required in later stages, for which the disease and the lesion should be "stable." We have encountered several cases of failure of vitiligo surgery, despite the patient's meeting the clinical criterion of stability of lack of any new lesions and/or worsening of existing lesions for at least 6 months to 2 years. Some of us (S.S., A.K.J., A.L.) have recently reported certain dermoscopic features highly suggestive of stability of vitiligo lesions that may serve as valuable adjuvant markers in qualification of the patient as a suitable candidate for surgery $[17,18]$. In our observation, perifollicular depigmentation, marginal hyperpigmentation, and presence of leukotrichia are suggestive of lesional stability (Figure 3A). An altered pigment pattern, perifollicular pigment retention, and certain specific features such as starburst appearance, comet tail sign, and "tapioca sago" appearance (Figure 3B) are suggestive of disease activity $[17,18]$. More recently, in vitiligo lesions treated surgically with micropunch grafting, we have described dermoscopic features that may serve as possibly the earliest prognostic markers of surgical outcome [19]. Another example is that of perifollicular erythema on trichoscopy as a well-established marker of active/ 
progressive disease in frontal fibrosing alopecia [20-22] (Figure 4).

\section{Defining Adequate Margins for Surgical Excision of Tumors}

Akin to ex-vivo dermoscopy, which is useful for the histopathologist, in-vivo dermoscopy can add to clinical examination for the operating surgeon during surgical excision of tumors such as the basal cell carcinoma (BCC). When surgical ablation is contemplated for a BCC, excision margins between 3 and $10 \mathrm{~mm}$ allow for radical excision in up to $95 \%$ of cases depending on the tumor site, size, borders, and histology. In a prospective study, 200 consecutive BCCs of the head and neck were removed with 2-mm dermoscopically detected excision margins that yielded histologically confirmed complete excisions in all but 3 cases $(197 / 200=98.5 \%)$ [23]. In another prospective case-control study that comprised 44 patients who underwent Mohs micrographic surgery, the group in which surgical margins were guided by dermoscopy demonstrated an outcome that was similar to that of the control group, suggesting that further research is warranted before recommending dermoscopy as an aid in demarcating surgical margins for Mohs micrographic surgery [24].

\section{Early Assessment of Therapeutic Response and Disease Stabilization}

The conventional method of evaluation of response to treatment involves interrogating the patient about symptomatic improvement and examining the skin for the extent and morphology of lesions. It is a hard fact that clinically appreciable improvement in many chronic skin disorders, especially pigmentary dermatoses and alopecias, often requires weeks to months. This often translates into a challenging situation for the treating dermatologist with respect to making a decision on continuing or changing the treatment protocol. This also results in patient dissatisfaction, noncompliance with further therapy, and "doctor hopping." Dermoscopy may serve as a

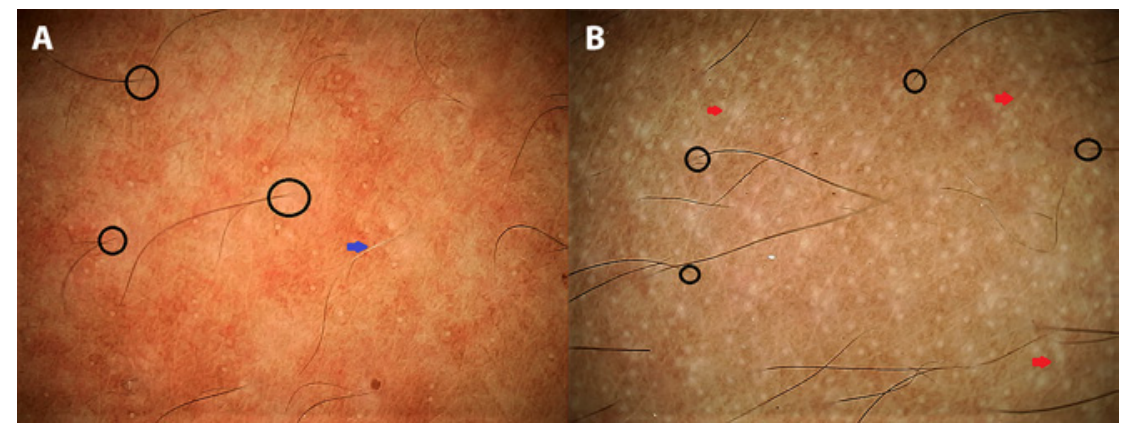

Figure 3. Application of dermoscopy in suggesting disease activity in vitiligo. (A) A stable lesion of vitiligo fit for surgery displaying perifollicular depigmentation (black circles) and leukotrichia (blue arrow). (B) An unstable lesion displaying altered pigment network, "tapioca sago" appearance with multiple pearly-white dots (larger than eccrine openings), and micro-Köbner phenomenon (red arrows). (Escope video dermatoscope, Timpac Healthcare Pvt. Ltd., New Delhi, India; polarized, ×20.) [Copyright: (C2019 Sonthalia et al.]

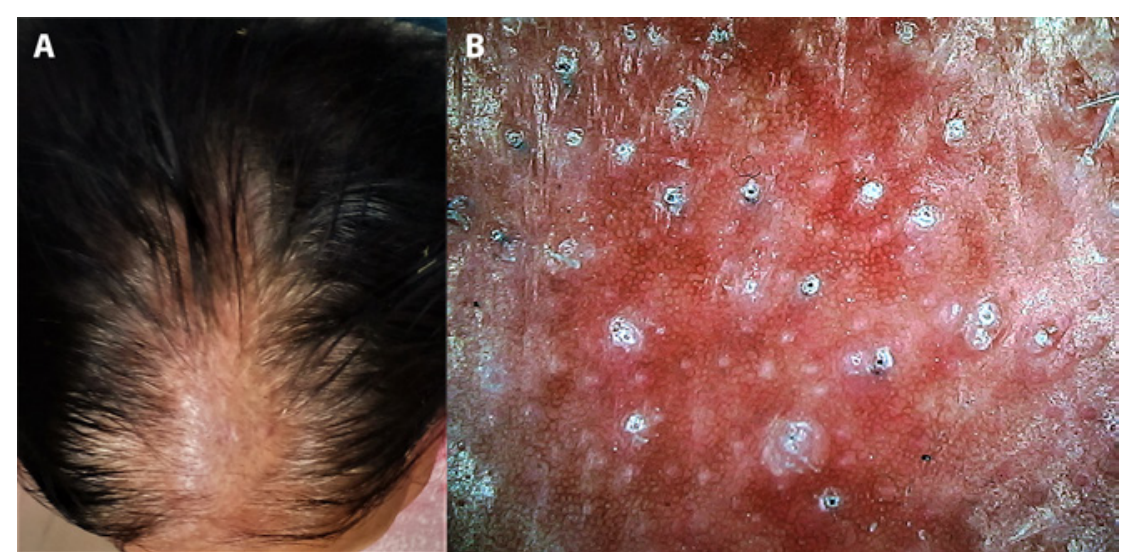

Figure 4. Application of dermoscopy in suggesting disease activity in frontal fibrosing alopecia. (A) Clinical image of suspected frontal fibrosing alopecia in an elderly woman. (B) Dermoscopic features of multiple large white dots (fibrosed follicular ostia) with perifollicular scales and casts confirming the diagnosis and prominent perifollicular erythema suggestive of active disease. (Escope video dermatoscope, Timpac Healthcare Pvt. Ltd., New Delhi, India; polarized, $\times 20$.) [Copyright: (C2019 Sonthalia et al.]

savior in such challenging situations, as the dermoscopic improvement tends to precede clinical improvement [1].

In alopecia areata (AA) for example, dermoscopy can confidently predict disease activity following treatment. Active AA shows black dots, "exclamation mark" or tapering hairs, broken hairs, yellow dots, and short vellus hairs (Figure 5A), whereas in a treatment-responsive patch of AA, black dots tend to disappear, pigtail and upright regrowing hairs appear, and yellow dots persist [11,25-27] (Figure 5B). Thus, trichoscopic review of AA patches being treated with regular intralesional steroid can help the clinician decide on the need for further sessions of intralesional steroid [27].

This application of dermoscopy has been documented to be useful in disorders of hyperpigmentation with either a relapsing-remitting course such as melasma [28] or a very slowly and poorly responsive course such as lichen planus pigmentosus [29]. In melasma, a recently published study as well as our own experience has shown that the presence of a prominent vascular component on dermoscopy (widened vessels) is a reliable indicator of expected improvement with vascular therapies such as pulsed-dye laser [30] and oral tranexamic acid (Figure 6). 


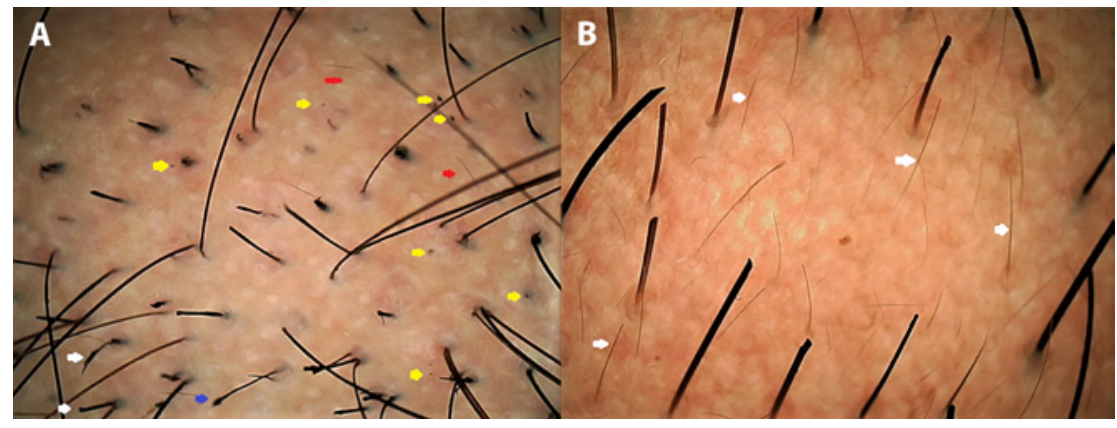

Figure 5. Dermoscopy in evaluating treatment response with disease activity. (A) Alopecia areata patch over the beard before treatment displaying a patch of alopecia with multiple black dots (yellow arrows) and a few yellow dots, short vellus hairs (red arrows), tapering hair (blue arrow), and dystrophic hairs (white arrows). (B) An adjacent patch after 2 sessions of intralesional triamcinolone displaying hair growth, dramatic reduction of black dots, persistent yellow dots, and multiple short up-growing hairs (white arrows) suggestive of good treatment response. (Escope video dermatoscope, Timpac Healthcare Pvt. Ltd., New Delhi, India; polarized, $\times 20$.) [Copyright: $@ 2019$ Sonthalia et al.]
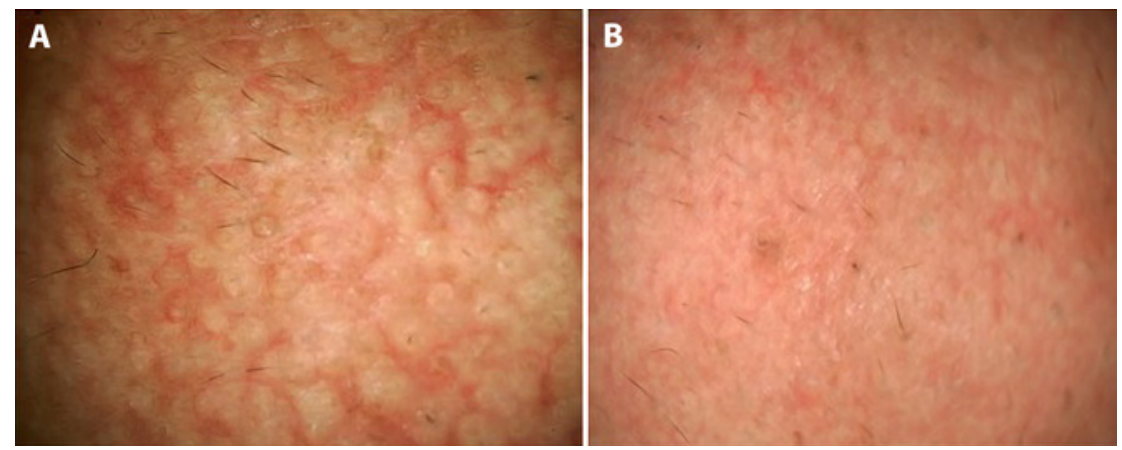

Figure 6. Dermoscopy in early demonstration of treatment response in melasma. (A) Dermoscopic image from an untreated macule of melasma over the cheek of a woman displaying a diffuse nonspecific pattern of dark brown pigmentation with plentiful scattered dark brown globules and clods with perifollicular sparing and multiple prominent telangiectasias. (B) After 30 days of nightly local application of a $2 \%$ hydroquinone cream and daytime sunscreen and oral tranexamic acid ( $250 \mathrm{mg}$ twice daily), dermoscopy from the same spot showing dramatic lightening of the background hue and erythema and reduction of pigmented structures and the telangiectasias. (Escope video dermatoscope, Timpac Healthcare Pvt. Ltd., New Delhi, India; polarized, $\times 20$.) [Copyright: $@ 2019$ Sonthalia et al.]

Dermoscopic reevaluation of the baseline lesion(s) on the subsequent visit not only allows the clinician to appreciate posttreatment improvement (or lack thereof), but provides reassurance to the patient about the commencement of improvement, thereby maintaining treatment compliance. This use of dermoscopy is not limited to only some disorders or any particular skin type, as it has been reported from various regions and for various disorders [3042], a few of which are enumerated in Table 3. Other than the evaluation of disease activity and therapeutic response, certain dermoscopic criteria (eg, pigmented structures, ulceration, and arborizing vessels in superficial BCCs treated with nonablative modalities) have been suggested to predict the presence of residual disease (residual disease-associated dermoscopic criteria) [43].

The aforesaid contention deepens due to the high prevalence $(3 \%-53.6 \%)$ of dysmorphophobia or body dysmorphic disorder in patients seeking clinical dermatology consultation as well as cosmetic procedures [44,45]. Although psychiatric/psychological intervention constitutes their primary treatment modality, ensuring their dermatological treatment compliance is vital until their psychiatric comorbidity improves. In our experience, the majority of such patients at least acknowledge the objective improvement appreciable on dermoscopy, which reinforces treatment compliance while the psychiatric treatment continues.

\section{Enhancing Accuracy of Reading Patch Test and Pathergy Test Results}

Reading allergy patch test reactions can be challenging in the dark skin, even with a hand lens. In doubtful reactions, we have found dermoscopic imaging to be helpful in confirmation of the reaction being true positive or irritant (Figure 7). Dermoscopy has also proved useful in the confirmation of pathergy test results in patients with Behçet disease [46].

\section{Therapeutic Efficacy Evaluation in Clinical Studies}

The satisfaction of many clinicians with dermoscopic images serving as an early, objective, and reliable measure of preand posttreatment comparison has culminated in the use of dermoscopy as an important ancillary tool for efficacy evaluation of existing as well as novel therapeutic modalities in clinical studies [30-42,47-50]. While the avoidance of or difficulty in repeating skin biopsy (whether due to patient noncompliance or due to the posttreatment unremarkability of the lesional morphology rendering biopsy-site selection difficult) constitutes one of the important reasons for the increasing use of dermoscopy and other noninvasive imaging techniques in therapeutic efficacy evaluation in clinical studies, in studies involving both histological and dermoscopic evaluation, the objective served is the concomitant establishment and validation of dermoscopic criteria for use in future studies that may not warrant histological confirmation. 
Table 3. List From Published Literature on the Use of Dermoscopy for Posttreatment Improvement Evaluation in Some Skin and Appendageal Disorders [30-42]

\begin{tabular}{|c|c|c|}
\hline Condition & $\begin{array}{c}\text { Treatment Administered or } \\
\text { Evaluated }\end{array}$ & Remarks \\
\hline \multirow[t]{2}{*}{ Melasma } & $\begin{array}{l}\text { 1,064-nm Q-switched Nd:YAG } \\
\text { (QSNY) laser vs QSNY + pulsed-dye } \\
\text { laser (PDL) }\end{array}$ & \\
\hline & 17 patients (split-face study) & \\
\hline Actinic keratosis & Ingenol mebutate $0.015 \%$ gel & 52 patients \\
\hline $\begin{array}{l}\text { Seborrheic } \\
\text { keratosis }\end{array}$ & $\begin{array}{l}\text { Novel aqueous solution containing } \\
\text { nitric acid, zinc and copper salts, and } \\
\text { organic acids }\end{array}$ & $\begin{array}{l}15 \text { patients with } \\
50 \text { seborrheic } \\
\text { keratoses }\end{array}$ \\
\hline $\begin{array}{l}\text { Male androgenetic } \\
\text { alopecia }\end{array}$ & $\begin{array}{l}\text { Platelet-rich plasma therapy }+ \\
\text { microneedling }\end{array}$ & 20 patients \\
\hline $\begin{array}{l}\text { Pigmented basal } \\
\text { cell carcinoma }\end{array}$ & Imiquimod $5 \%$ cream & 20 lesions \\
\hline Plaque psoriasis & $\begin{array}{l}\text { Biological therapy: any of the } 4 \\
\text { agents (adalimumab, etanercept, } \\
\text { infliximab, and ustekinumab) }\end{array}$ & 75 patients \\
\hline $\begin{array}{l}\text { Verrucous } \\
\text { epidermal nevus }\end{array}$ & $\begin{array}{l}\text { Pulsed CO2 laser vs erbium:YAG } \\
\text { laser }\end{array}$ & $\begin{array}{l}20 \text { patients ( } 10 \text { in } \\
\text { each group) }\end{array}$ \\
\hline Bowen disease & $\begin{array}{l}\text { Imiquimod } 5 \% \text { cream OR } \\
\text { photodynamic therapy }\end{array}$ & $\begin{array}{l}23 \text { patients with } \\
29 \text { lesions }\end{array}$ \\
\hline $\begin{array}{l}\text { Vulvar lichen } \\
\text { sclerosus }\end{array}$ & $\begin{array}{l}\text { Mometasone furoate } 0.1 \% \\
\text { ointment + tretinoin } 0.05 \% \text { cream } \\
\text { in short-contact therapy (group } \\
\text { A) vs mometasone furoate } 0.1 \%+ \\
\text { emollient (group B) }\end{array}$ & 32 patients \\
\hline Vitiligo (localized) & $\begin{array}{l}\text { Combination therapy of tacrolimus } \\
\text { ointment }+308 \text {-nm excimer laser }\end{array}$ & 147 patients \\
\hline $\begin{array}{l}\text { Axillary hair } \\
\text { removal }\end{array}$ & $\begin{array}{l}\text { Diode laser vs intense pulsed light } \\
\text { (IPL) }\end{array}$ & $\begin{array}{l}21 \text { patients (split- } \\
\text { half protocol) }\end{array}$ \\
\hline $\begin{array}{l}\text { Facial/upper limb } \\
\text { telangiectasias } \\
\text { associated with } \\
\text { systemic sclerosis }\end{array}$ & PDL vs IPL & $\begin{array}{l}19 \text { patients (split- } \\
\text { half protocol) }\end{array}$ \\
\hline Scabies & $\begin{array}{l}\text { Foam containing } 0.165 \% \text { pyrethrins } \\
\text { and } 1.65 \% \text { piperonyl butoxide }\end{array}$ & 20 patients \\
\hline
\end{tabular}

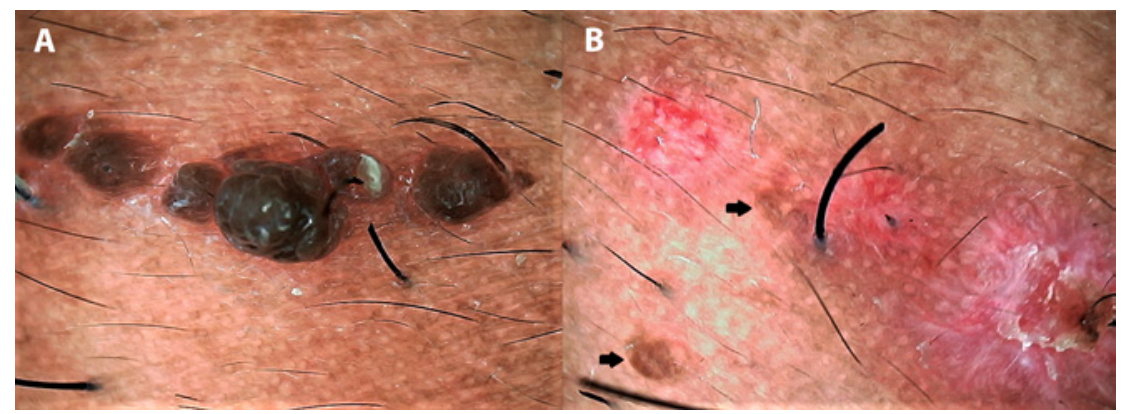

Figure 8. Dermoscopy for cosmetic wart mapping in darker skin. (A) Linear köbnerized cluster of verruca barbae in an Indian man before ablation. (B) Dermoscopy done after radiofrequency ablation of the warts (erosions) revealing at least 2 warts (black arrows) missed despite using magnifying surgical loupes during the procedure. (Escope video dermatoscope, Timpac Healthcare Pvt. Ltd., New Delhi, India; polarized, ×20.) [Copyright: (C2019 Sonthalia et al.]

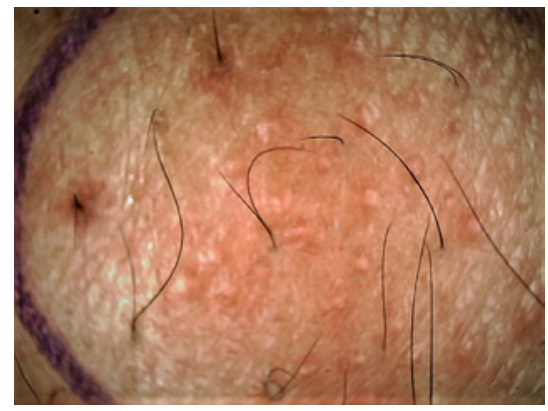

Figure 7. Dermoscopic aid in confirming patch test results. A grossly faint positive reaction displayed erythema and papulovesicles on dermoscopy confirming grade 2 patch test positivity. (Escope video dermatoscope, Timpac Healthcare Pvt. Ltd., New Delhi, India; polarized, $\times 20$.) [Copyright: C2019 Sonthalia et al.]

\section{Expanding the Realm of Dermoscopy}

\section{"Cosmetic Wart Mapping"}

Akin to "mole-mapping" in the whiteskinned population, video dermoscopic scanning can be useful for mapping of multiple cosmetic pigmented warts (usually verruca plana [VP]) in darkskinned patients, particularly involving the beard area in men and periorbital area in women. Cosmetic warts arise from a single or few verrucae over the face due to pseudoköbnerization induced by temporary hair removal methods such as shaving, threading, and waxing [51]. Men with multiple VP of the beard region or women with VP involving the threading-treated areas around the eyebrows can be provided with ablation of many more lesions if video dermoscopy is used to scan the affected area compared to the conventional ablation of lesions visible to the unaided eye (Figure 8). If left untreated, these tiny lesions invisible to the eye and hand lens might contribute to further dissemination $[1,2,28]$.

\section{Aesthetic Applications of Dermoscopy}

Dermoscopy also finds its utility in certain cosmetic/aesthetic procedures. A dermoscopic photo-aging scale has been recently conceived and found to be a 


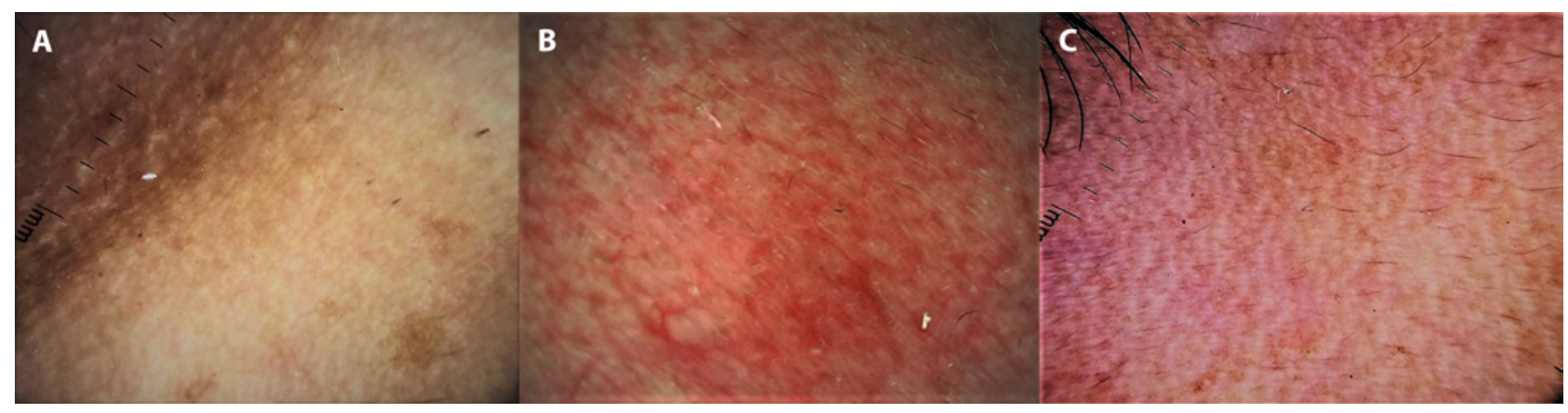

Figure 9. Dermoscopy of the under-eye region for management of periocular pigmentation. (A) Predominantly pigmentary component; (B) predominantly vascular component; and $(\mathrm{C})$ combination of pigmentary and vascular component. (DermLite DL4, 3 Gen Inc., USA; polarized, $\times 10$.) [Copyright: $\odot 2019$ Sonthalia et al.]

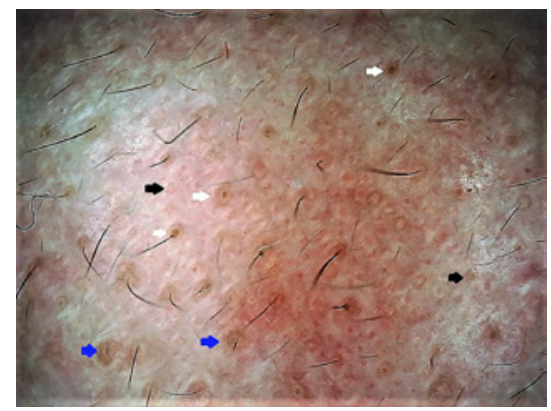

Figure 10. Dermoscopy of skin pores. Well-defined pilosebaceous openings or pores visible on dermoscopy of the cheek of an Indian woman with enlarged open pores. Note the presence of visible skin pores (black arrows), enlarged skin pores (blue arrows), and blackhead-embedded skin pores (white arrows). (Escope video dermatoscope, Timpac Healthcare Pvt. Ltd., New Delhi, India; polarized, $\times 20$.) [Copyright: (C2019 Sonthalia et al.]

reliable and valid diagnostic tool for quantitative evaluation of photo-aged facial skin in a white population and to study the effect of preventive and therapeutic approaches to skin aging in the future [52]. Dermoscopic evaluation of periocular hyperpigmentation and under-eye bags can provide a clue to the predominant abnormality (skin pigmentation, abnormal vasculature, skin laxity) (Figure 9) and aid in development of customized treatment protocols. In a recent study evaluating the impact of normobaric oxygen therapy on periorbital darkening, dermoscopy was used as the main tool to document posttherapy improvement and revealed a substantial reduction in vascularity and pigmentation with more than a quarter of patients showing $>50 \%$ improvement [53].

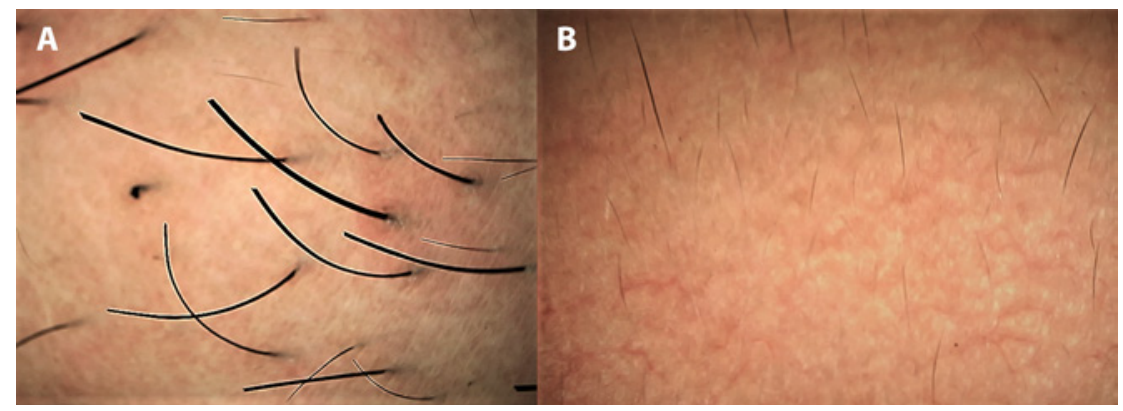

Figure 11. Dermoscopy for deciding laser/light device and parameters for permanent hair reduction. (A) Dermoscopy of the chin of an obese woman with polycystic ovarian syndrome revealing thick terminal hairs and darkly pigmented skin. (B) Image from the same patient after 8 sessions of diode laser (with low energy) showing dramatic scattering and thinning of chin hairs. (Escope video dermatoscope, Timpac Healthcare Pvt. Ltd., New Delhi, India; polarized, $\times 20$.) [Copyright: $@ 2019$ Sonthalia et al.]

Enlarged facial skin pores is a common cosmetic complaint, especially in individuals with oily skin. Dermoscopy can aid in evaluating and defining "enlarged" open facial pores [54]. Kim et al have defined and categorized the morphological subtypes of skin pores into 3 different categories: "visible skin pores" (0.1-0.6 $\left.\mathrm{mm}^{2}\right)$, "enlarged skin pores" $\left(0.3-0.6 \mathrm{~mm}^{2}\right)$, and "blackhead embedded skin pores" using the Robo Skin Analyzer CS50 (NIIC, Tokyo, Japan), which has a dermatoscope-like attachment mode in addition to other functions [55]. Figure 10 provides a gross idea of types of pores on a standard dermoscopic evaluation.

Selecting the right parameters while using devices for laser hair reduction is not only crucial for optimum outcome and patient satisfaction, it is critical to prevent laser-induced burn and postprocedure hyperpigmentation in dark- skinned individuals (Fitzpatrick skin photo types IV-VI). In our own experience, dermoscopic evaluation of the scattering and thickness of hairs before the laser session has proven useful in dark-skinned patients with hirsutism. Optimal laser parameters (especially pulse duration that depends on the thickness of the hair being targeted) can thus be correspondingly fine-tuned. Moreover, apprehensive patients can be shown the laser-induced scattering and thinning of hirsute hairs to maintain or improve compliance (Figure 11). In fact, a recent study has emphasized the utility of trichoscopy in monitoring the response to laser hair reduction in women [56].

Trichoscopy is of great value to a hair transplant surgeon. It aids in recording the number of existing follicular units, the number of hairs per follicular unit, the size of hair follicles, as well 
as the interfollicular distance at both the donor and recipient site. In addition, trichoscopy may be useful for early differential diagnosis of posttransplant surgical complications such as folliculitis and secondary lichen planopilaris. A detailed account of clinco-trichoscopic correlation in the scalp of patients who underwent hair transplantation has been documented by Madura et al [57].

\section{Detection of Intra/Subcutaneous Foreign Bodies and Retained Sutures}

The detection and removal of retained sutures in a crusted wound may sometimes become daunting, especially when black threads (typically silk) are used in individuals with darker skin due to the relative lack of color contrast compared with fair skin. In such situations, dermoscopic assessment provides an excellent 3-dimensional view that may facilitate their prompt identification and removal (Figure 12) [28]. A blue Prolene suture should help in avoiding this issue in all skin types, although dermoscopy may still help in visualizing the ends of the residual suture if stitch removal is done incorrectly. Dermoscopy has also been documented for its application in facilitating identification and removal of a foreign body lodged in the skin (Figure 13) $[28,58]$.

\section{Teledermoscopy}

Teledermatology is a subspecialty of dermatology that uses telecommunication technologies to transfer medical information over varying distances. The format can be audio, visual, and/or data-based. Digital dermoscopy refers to the acquisition and storage of digital images from a dermoscopic examination. Teledermoscopy is the transferring of a digitalized dermoscopic image (visual data) for diagnosis (Figure 14), education, consultation, or follow-up. Mobile teledermoscopy uses a smartphone to deliver the same type of service [59]. These images are also used in machine learning [60,61], a rapidly

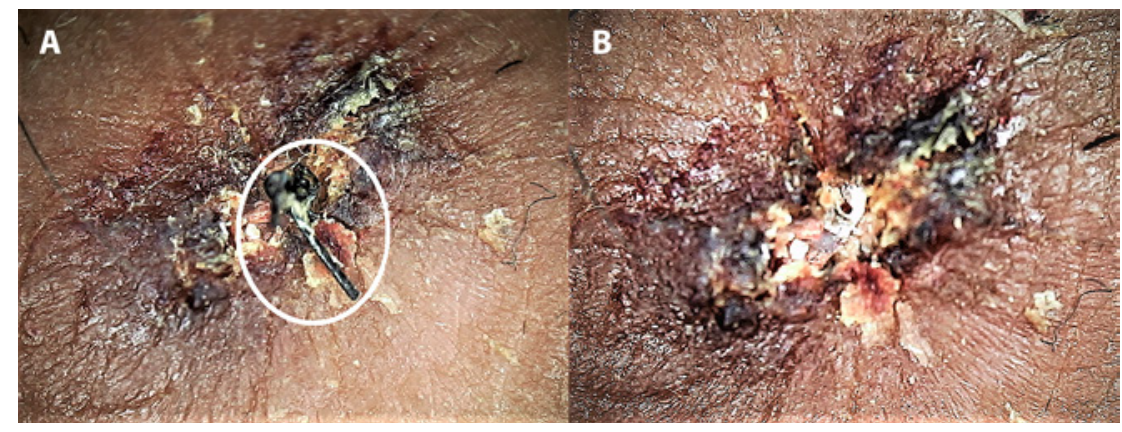

Figure 12. Dermoscopy for identification and removal of retained sutures in a heavily crusted wound. (A) Before removal, the black suture, which was otherwise extremely difficult to delineate from the surrounding brownish black crust, is easily visible on dermoscopy (white circle). (B) After removal. (Escope video dermatoscope, Timpac Healthcare Pvt. Ltd., New Delhi, India; polarized, ×20.) [Copyright: @2019 Sonthalia et al.]

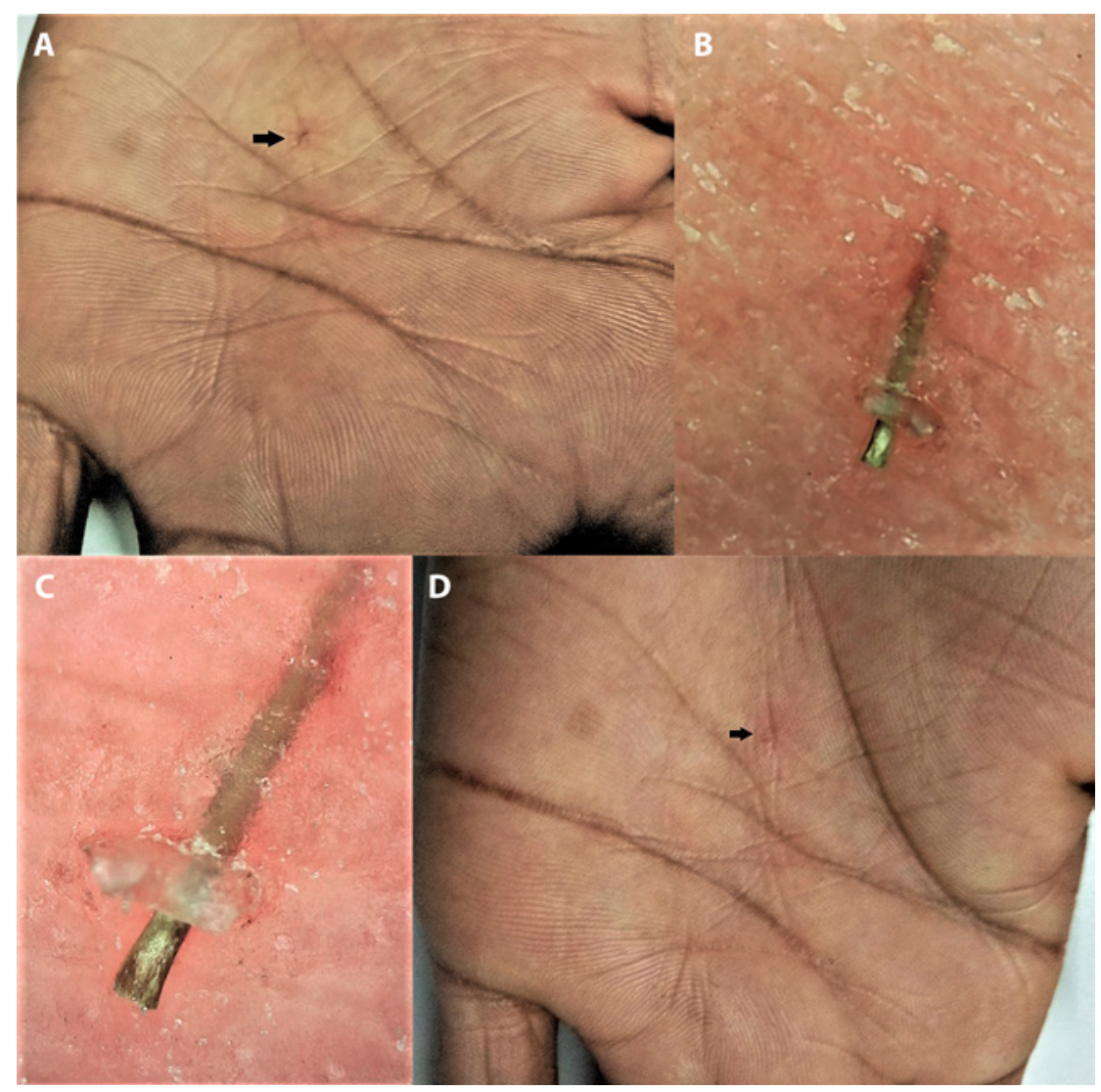

Figure 13. Dermoscopy for identification and removal of intracutaneous foreign body. (A) Clinical image before removal-the tiny foreign body lodged intracutaneously (black arrow) with no visibility of protruding head or tail for removal. (B) Low magnification $(\times 20)$ polarized dermoscopic image. (C) High magnification $(\times 70)$ polarized dermoscopic image revealing the protruding head-end of the foreign body. (Escope video dermatoscope, Timpac Healthcare Pvt. Ltd., New Delhi, India; polarized, ×20.) (D) Clinical image after dermoscopically facilitated removal revealing a tiny linear subcorneal erosion (black arrow). [Copyright: C2019 Sonthalia et al.]

developing area that will soon change our way of seeing and understanding a new device or medication.

Teledermoscopy's greatest strength may be as a triage and monitoring tool, as it can reduce the number of unneces- sary referrals, wait times, and the cost of providing and receiving dermatological care [61]. Inclusion of dermoscopic images in teledermatology-based consultations is known to improve decisions on triaging and surgical excision of mela- 


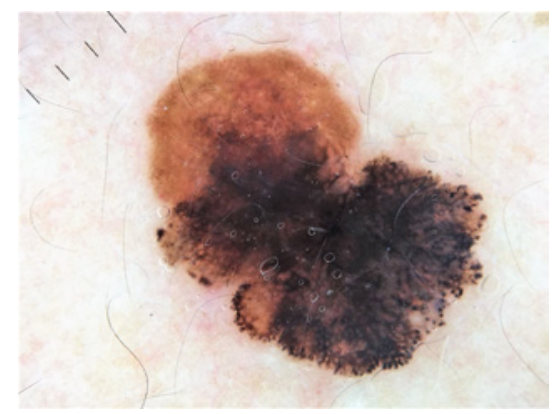

Figure 14. Dermoscopic image received via teledermatology consultation with a family physician. The patient was referred directly to surgery, and the diagnosis of melanoma was confirmed by pathology. (DermLite DL4, 3 Gen Inc., USA; polarized, $\times 10$.) [Copyright: (C2019 Sonthalia et al.]

nomas and other skin cancers [59]. The concept of teledermoscopy has futuristic implications for health care systems in the developing world [61], where a large proportion of dermatology patients are still managed by primary care physicians [62]. Of course, this goal can be successfully achieved only with proper training into dermoscopy and principles of teledermoscopy of both primary care physicians and dermatologists.

\section{Psychological Realm of Dermoscopy}

Dermoscopy: The Dermatologist's Tool in the Era of Social Media Empowerment of Patients

In the current era, the practice of medicine and specifically dermatology is facing a new challenge. Over and above the chronicity and relapsing nature of the majority of cutaneous disorders, the impact of health-related information available on the internet and exchanged on social media has dramatically altered the approach to practicing dermatology [63]. While a detailed discussion on the impact of social media on the practice of dermatology is beyond the scope of this article, it may suffice to mention that the above concerns make a strong point favoring the concept of "e-professionalism" as a distinct new paradigm requiring particular training, practice, and formulation of new policies that prescribe guidelines for normative behavior to regulate and reduce social media use in dermatology [64]. Pending global and regional consensus guidelines on regulating the use of social media in health care, dermoscopy may serve as an efficient tool for counteracting the invalid and illogical interjections of the "google generation" patients in the diagnostic and therapeutic decisions made by dermatologists.

\section{Dermatoscope: A Professional Highlight for Dermatologists}

While a hand lens, corneal loupe, and other conventional tools of magnification are present in nearly every dermatologist's office, a dermatoscope definitely adds to the professional standing. Patients tend to place more faith in a dermatologist who is well-versed in dermoscopy and uses it not just for making diagnoses, but also to counsel them on pertinent features appreciable on dermoscopy that are of relevance to their understanding and improved therapeutic compliance.

\section{Dermatoscope: The Dermatologist's Stethoscope}

Cutaneous disorders are often chronic, treatment-recalcitrant, and with an unpredictable/relapsing-remitting course. Patients with conditions such as psoriasis, vitiligo, nonremitting AA, and chronic eczemas typically consult different dermatologists over time, hoping for a permanent cure. Chronic skin diseases may have a devastating effect on a person's physical and psychological well-being, affecting his or her education, relationships, career choices, social and leisure activities, and sex life. The adverse physical, psychological, and social consequences of chronic dermatoses affect not only the patient, but also caregivers and family members [65]. Demonstration of dermoscopic images (in select cases) to the patient and/or relatives tends to improve the patient's psychodermatology profile and provides reassurance to caregivers [1,2]. The relation between a patient's psychological well-being and response to therapy need not be overemphasized. Dermoscopic examination and counseling thereof tend to make such patients and family members feel they are being cared for.

\section{Future Perspective}

Innovations in the technique and utilization of dermoscopy for better imaging and expanding its extradiagnostic application are being conceived and published at a fast pace. One such newfangled approach is dermoscopy-guided darkfield multifunctional optical coherence tomography, conceptualized and developed by Kwon et al [66]. By combining the 2 imaging techniques, Kwon et al achieved simultaneous high-contrast superficial information (dermoscopy) and depth-resolved structural, birefringent, and vascular information of the skin (multifunctional optical coherence tomography) and documented its utility in better overall evaluation of different skin lesions.

Akin to endoscopic procedures in surgical branches, we believe that the dermatoscope may be amenable to redesigning with creation of multiple portals for diagnostic and therapeutic interventions [67] delivered through the device to allow high-precision diagnostic and dermatosurgical procedures under concomitant dermoscopic visualization.

\section{Conclusions}

Dermoscopy has far-reaching uses beyond just diagnosis of skin, hair, and nail disorders. Some can be highly relevant for both physicians and patients, offering prompt resolution to diagnostic and therapeutic dilemmas encountered routinely. The quest for more uses of this technique continues and mastering this technology becomes imperative for not only dermatologists, but also primary care physicians, pediatricians, and specialists of other branches of medicine. 


\section{References}

1. Sonthalia S, Errichetti E. Dermoscopy—not just for diagnosis and not just for dermatologists! Kathmandu Univ Med J (KUMJ). 2017;15(1):1-2.

2. Sonthalia S, Kaliyadan F. Dermoscopy Overview and Extradiagnostic Applications. [Updated 2019 Jan 20]. In: StatPearls [Internet]. Treasure Island (FL): StatPearls Publishing; 2019 Jan2019 May 11. . Available at: https://www.ncbi.nlm.nih.gov/books/ NBK537131/. Accessed on May 13, 2019.

3. Errichetti E, Stinco G. Dermoscopy in general dermatology: a practical overview. Dermatol Ther (Heidelb). 2016;6(4):471-507.

4. Watanabe S, Sawada M, Dekio I, Ishizaki S, Fujibayashi M, Tanaka M. Chronology of lichen planus-like keratosis features by dermoscopy: a summary of 17 cases. Dermatol Pract Concept. 2016;6(2):29-35.

5. Mirmirani P, Willey A, Headington JT, Stenn K, McCalmont TH, Price VH. Primary cicatricial alopecia: histopathologic findings do not distinguish clinical variants. J Am Acad Dermatol. 2005;52(4):637-643.

6. Thakur BK, Verma S, Raphael V. Clinical, trichoscopic, and histopathological features of primary cicatricial alopecias: a retrospective observational study at a tertiary care centre of North East India. Int J Trichology. 2015;7(3):107-112.

7. Ankad BS, Beergouder SL, Moodalgiri VM. Lichen planopilaris versus discoid lupus erythematosus: a trichoscopic perspective. Int J Trichology. 2013;5(4):204-207.

8. Gálvez-Canseco A, Sperling L. Lichen planopilaris and frontal fibrosing alopecia cannot be differentiated by histopathology. $J$ Cutan Pathol. 2018;45(5):313-317.

9. Trotter MJ, Au S, Naert KA. Practical strategies to improve the clinical utility of the dermatopathology report. Arch Pathol Lab Med. 2016;140(8):759-765.

10. Miteva M, Tosti A. Dermoscopy guided scalp biopsy in cicatricial alopecia. J Eur Acad Dermatol Venereol. 2013;27(10):1299-1303.

11. Lacarrubba F, Micali G, Tosti A. Scalp dermoscopy or trichoscopy. Curr Probl Dermatol. 2015;47:21-32.

12. Liu WC, Tey HL, Lee JS, Goh BK. Exogenous ochronosis in a Chinese patient: use of dermoscopy aids early diagnosis and selection of biopsy site. Singapore Med J. 2014;55(1):e1-e3.

13. Choo JY, Bae JM, Lee JH, Lee JY, Park YM. Blue-gray blotch: a helpful dermoscopic finding in optimal biopsy site selection for true vasculitis. J Am Acad Dermatol. 2016;75(4):836-838.

14. Bet DL, Reis AL, Di Chiacchio N, Belda Junior W. Dermoscopy and onychomycosis: guided nail abrasion for mycological samples. An Bras Dermatol. 2015;90(6):904-906.

15. Merkel EA, Amin SM, Lee CY, et al. The utility of dermoscopy-guided histologic sectioning for the diagnosis of melanocytic lesions: a case-control study. J Am Acad Dermatol. 2016;74(6):1107-1113.

16. Haspeslagh M, Hoorens I, Degryse N, et al. Pathologic evaluation of skin tumors with ex vivo dermoscopy with derm dotting. JAMA Dermatol. 2017;153(2):154-161.

17. Jha AK, Sonthalia S, Lallas A, Chaudhary RKP. Dermoscopy in vitiligo: diagnosis and beyond. Int J Dermatol. 2018;57(1):50-54.

18. Jha AK, Sonthalia S, Lallas A. Dermoscopy as an evolving tool to assess vitiligo activity. J Am Acad Dermatol. 2018;78(5):10171019.

19. Jha AK, Sonthalia S, Lallas A, Chaudhary RKP. Post-graft trichrome and Manchurian gravy signs on dermoscopy can predict disease activity in vitiligo lesions post-skin grafting. Int J Dermatol. 2018;57(11):e144-e145.

20. Toledo-Pastrana T, Hernández MJ, Camacho Martínez FM. Perifollicular erythema as a trichoscopy sign of progression in frontal fibrosing alopecia. Int J Trichology. 2013;5(3):151-153.

21. Lis-Święty A, Miziołek B, Ranosz-Janicka I, Bierzyńska-Macyszyn G, Brzezińska-Wcisło L. Thermal imaging and dermoscopy for detecting inflammation in frontal fibrosing alopecia. $J$ Cosmet Dermatol. 2018;17(2):268-273. Epub 2017 Jul 20.

22. Sonthalia S, Jha AK, Tiwary PK. A dermoscopic diagnosis and activity evaluation of frontal fibrosing alopecia in an Indian lady. Indian Dermatol Online J. 2017;8(2):162-163.

23. Caresana G, Giardini R. Dermoscopy-guided surgery in basal cell carcinoma. J Eur Acad Dermatol Venereol. 2010;24(12):13951399.

24. Suzuki HS, Serafini SZ, Sato MS. Utility of dermoscopy for demarcation of surgical margins in Mohs micrographic surgery. An Bras Dermatol. 2014;89(1):38-43.

25. Jain N, Doshi B, Khopkar U. Trichoscopy in alopecias: diagnosis simplified. Int J Trichology. 2013;5(4):170-178.

26. Kibar M, Aktan Ş, Lebe B, Bilgin M. Trichoscopic findings in alopecia areata and their relation to disease activity, severity and clinical subtype in Turkish patients. Australas J Dermatol. 2015;56(1):e1-e6.

27. Ganjoo S, Thappa DM. Dermoscopic evaluation of therapeutic response to an intralesional corticosteroid in the treatment of alopecia areata. Indian J Dermatol Venereol Leprol. 2013;79(3):408417.

28. Sonthalia S, Kaur T, Srivastava S. Monitoring of therapeutic response and other applications. In: Lallas A, Errichetti E, Ioannides D, eds. Dermoscopy in General Dermatology. Boca Raton, FL: CRC Press; 2018:325-329.

29. Sonthalia S, Vedamurthy M, Thomas M, et al. Modified phenol peels for treatment-refractory hyperpigmentation of lichen planus pigmentosus: a retrospective clinico-dermoscopic analysis. J Cosmet Dermatol. Epub 2019 Jan 20. https://doi.org/10.1111/ jocd.12862.

30. Kong SH, Suh HS, Choi YS. Treatment of melasma with pulseddye laser and1,064-nm Q-switched Nd:YAG laser: a split-face study. Ann Dermatol. 2018;30(1):1-7.

31. Carbotti M, Coppola R, Zanframundo S, Devirgiliis V, Panasiti V. Efficacy of ingenol mebutate in the treatment of actinic keratoses: a pre- and posttreatment dermoscopic comparative analysis. Biomed Res Int. 2018;2018:4381019.

32. Lacarrubba F, Nasca MR, Verzì AE, Micali G. A novel topical agent in the treatment of seborrheic keratoses: a proof of concept study by clinical and dermoscopic evaluation. Dermatol Ther. 2017;30(5):e12526.

33. Jha AK, Udayan UK, Roy PK, Amar AKJ, Chaudhary RKP. Platelet-rich plasma with microneedling in androgenetic alopecia along with dermoscopic pre- and post-treatment evaluation. J Cosmet Dermatol. 2018;17(3):313-318.

34. Husein-ElAhmed H, Fernandez-Pugnaire MA. Dermatoscopy-guided therapy of pigmented basal cell carcinoma with imiquimod. An Bras Dermatol. 2016;91(6):764-769.

35. Lallas A, Argenziano G, Zalaudek I, et al. Dermoscopic hemorrhagic dots: an early predictor of response of psoriasis to biologic agents. Dermatol Pract Concept. 2016;6(4):7-12.

36. Osman MAR, Kassab AN. Carbon dioxide laser versus erbium: YAG laser in treatment of epidermal verrucous nevus: a 
comparative randomized clinical study. J Dermatolog Treat. 2017;28(5):452-457.

37. Mun JH, Park JM, Song M, et al. The use of dermatoscopy to monitor therapeutic response of Bowen disease: a dermatoscopic pathological study. Br J Dermatol. 2012;167(6):1382-1385.

38. Corazza M, Maietti E, Toni G, Virgili A, Borghi A. Combining topical tretinoin with mometasone furoate in the treatment of vulvar lichen sclerosus: results of dermoscopic assessment. Dermatol Ther. 2018;31(6):e12735.

39. Wang LM, Lu WJ, Yuan JT, et al. Utility of dermoscopy for evaluating the therapeutic efficacy of tacrolimus ointment plus 308-nm excimer laser combination therapy in localized vitiligo patients. Exp Ther Med. 2018;15(4):3981-3988.

40. Ormiga P, Ishida CE, Boechat A, Ramos-E-Silva M. Comparison of the effect of diode laser versus intense pulsed light in axillary hair removal. Dermatol Surg. 2014;40(10):1061-1069.

41. Dinsdale G, Murray A, Moore T, et al. A comparison of intense pulsed light and laser treatment of telangiectases in patients with systemic sclerosis: a within-subject randomized trial. Rheumatology (Oxford). 2014;53(8):1422-1430.

42. Micali G, Lacarrubba F, Tedeschi A. Videodermatoscopy enhances the ability to monitor efficacy of scabies treatment and allows optimal timing of drug application. J Eur Acad Dermatol Venereol. 2004;18(2):153-154.

43. Apalla Z, Lallas A, Tzellos T, et al. Applicability of dermoscopy for evaluation of patients' response to nonablative therapies for the treatment of superficial basal cell carcinoma. $\mathrm{Br} J$ Dermatol. 2014;170(4):809-815.

44. Koblenzer CS. Body dysmorphic disorder in the dermatology patient. Clin Dermatol. 2017;35(3):298-301.

45. Kuhn H, Cunha PR, Matthews NH, Kroumpouzos G. Body dysmorphic disorder in the cosmetic practice. G Ital Dermatol Venereol. 2018;153(4):506-515.

46. Scherrer MA, de Castro LP, Rocha VB, Pacheco L. The dermatoscopy in the skin pathergy testing: case series in patients with suspected Behçet's disease [in Portuguese]. Rev Bras Reumatol. 2014;54(6):494-498.

47. Micali G, Dall’Oglio F, Verzì AE, Luppino I, Bhatt K, Lacarrubba F. Treatment of erythemato-telangiectatic rosacea with brimonidine alone or combined with vascular laser based on preliminary instrumental evaluation of the vascular component. Lasers Med Sci. 2018;33(6):1397-1400.

48. Huang Y, Zhuo F, Li L. Enhancing hair growth in male androgenetic alopecia by a combination of fractional $\mathrm{CO}_{2}$ laser therapy and hair growth factors. Lasers Med Sci. 2017;32(8):1711-1718.

49. Fabbrocini G, Panariello L, DE Padova MP, et al. Efficacy and tolerability of a spray product containing hydroxypropyl chitosan, climbazole and piroctone olamine, applied twice weekly for the treatment of the pitiriasis versicolor. G Ital Dermatol Venereol. 2017;152(6):565-568.

50. Grippaudo FR, Di Russo PP. Effects of topical application of B-resorcinol and glycyrrhetinic acid monotherapy and in combination with fractional $\mathrm{CO}_{2}$ laser treatment for benign hand hyperpigmentation treatment. J Cosmet Dermatol. 2016;15(4):413-419.
51. Sidharth S, Rahul A, Rashmi S. Cosmetic warts: pseudo-koebnerization of warts after cosmetic procedures for hair removal. J Clin Aesthet Dermatol. 2015;8(7):52-56.

52. Isik B, Gurel MS, Erdemir AT, Kesmezacar O. Development of skin aging scale by using dermoscopy. Skin Res Technol. 2013;19(2):69-74.

53. Mostafa WZ, Kadry DM, Mohamed EF. The effects of normobaric oxygen therapy on patients with periorbital darkening: an open, uncontrolled trial. Indian J Dermatol Venereol Leprol. 2015;81(4):427-429.

54. Uhoda E, Piérard-Franchimont C, Petit L, Piérard GE. The conundrum of skin pores in dermocosmetology. Dermatology. 2005;210(1):3-7.

55. Kim BY, Choi JW, Park KC, Youn SW. Sebum, acne, skin elasticity, and gender difference-which is the major influencing factor for facial pores? Skin Res Technol. 2013;19(1):e45-53.

56. Mohamed EE, Ahmed AM, Tawfik KM, Ibrahim SM. Trichoscopic changes in hair during treatment of hirsutism with 1064-nm neodymium:yttrium-aluminum-garnet laser. J Cosmet Dermatol. 2016;15(1):31-35.

57. Madura C, Ravipati N, Chandrashekhar BS. Clinical and trichoscopic correlation in scalp of patients who had undergone hair transplantation. International Journal of Dermoscopy. 2017;1(1):20-25.

58. Sonthalia S, Jha AK, Kaliyadan F. Dermoscopy for the detection and safe extraction of an intracutaneous foreign body. J Am Acad Dermatol. 2018;79(2):e19-e20.

59. Lee KJ, Finnane A, Soyer HP. Recent trends in teledermatology and teledermoscopy. Dermatol Pract Concept. 2018;8(3):214223.

60. Bleicher B, Levine A, Markowitz O. Going digital with dermoscopy. Cutis. 2018;102(2):102-105.

61. Pasquali P, Sonthalia S, Moreno DR, et al. Teledermatology and its current perspective. Indian Dermatol Online J. In press.

62. Sonthalia S, Agrawal M, Goldust M, Das S, Bhattacharya SN. Antifungal therapeutic failures in India: an important issue being overlooked. Lancet Infect Dis. 2018;18(11):1181-1182.

63. Sonthalia S, Arora D, Sharma A, Mhatre M. Role of social media in clinical practice: legal implications. In: Mysore M, Bhat S, Sirur SP, eds. Medicolegal Aspects of Dermatology \& Plastic Surgery. New Delhi: Jaypee Brothers Medical Publishers (P) Ltd; 2019:193-222.

64. Cain J, Romanelli F. E-professionalism: a new paradigm for a digital age. Curr Pharm Teach Learn. 2009;1(2):66-70.

65. Jafferany M, Pastolero P. Psychiatric and psychological impact of chronic skin disease. Prim Care Companion CNS Disord. 2018;20(2). pii: 17nr02247.

66. Kwon S, Yoon Y, Kim B, et al. Dermoscopy guided dark-field multi-functional optical coherence tomography. Biomed Opt Express. 2017;8(3):1372-1381.

67. Sonthalia S, Khurana R. Interventional Dermoscopy. J Am Acad Dermatol. 2019. Apr 5. pii: S0190-9622(19)30532-8. doi: 10.1016/j.jaad.2019.04.004. [Epub ahead of print] 